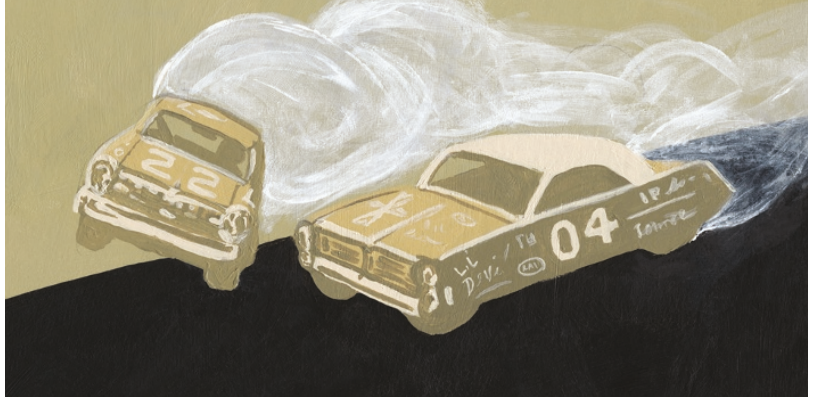

CHROMOSOMAL INSTABILITY

\section{Fast is dangerous}

Familial adenomatous polyposis is caused by an inherited mutation of adenomatosis polyposis coli $(A P C)$ and predisposes individuals to the development of colorectal polyps and colon cancer. $A p c^{+-}$mutant mice, however, have more polyps in the small intestine than in the colon. Aoki et al. now show that if the homeobox transcription factor gene $C d x 2$ - expression of which is reduced in most human colon cancers - is also mutated in these mice, the polyposis phenotype is similar to that found in humans. The authors show that disruption of $C d x 2$ causes fast transition through the G1-S phase of the cell cycle, leading to chromosomal instability and increased polyposis.

First, the authors observed that $A p c^{+/ \Delta 716} C d x 2^{+/-}$mice had more colon polyps than $A p c^{+/ \Delta 716}$ mice and that these polyps had reduced expression of $C d x 2$. The polyps in $A p c^{+/ \Delta 716} C d x 2^{+/-}$mice also showed loss of heterozygosity (LOH) at the Apclocus. In most Apc mouse models, $\mathrm{LOH}$ is caused by chromosomal instability and Aoki et al. showed that $C d x 2^{+/-}$and $A p c^{+/ \Delta 716} C d x 2^{+/-}$mice had higher chromosome instability than $A p c^{+/ \Delta 716}$ mice.

Next, Aoki et al. decided to fill in the gaps - how does reduction of $\mathrm{CDX} 2$ cause chromosomal instability? They observed that a human colon cancer cell line (DLD-1) with reduced CDX2 underwent an accelerated G1-S transition compared with DLD-1 cells with normal levels of CDX2. Because activation of the phosphatidylinositol 3-kinase (PI3K) pathway accelerates G1 progression, the authors examined proteins in this pathway and found that mammalian target of rapamycin (mTOR) a downstream effector of PI3K — was activated. Overexpression of CDX2 in DLD-1 cells suppressed mTOR activity and reduced the speed of the G1-S transition, confirming that mTOR activity is regulated by $\mathrm{CDX} 2$. In addition, expression of constitutively active AKT - an upstream effector of mTOR — led to high

chromosome instability in DLD-1 cells and in the colon polyp cells of $A p c^{+/ \Delta 716} \mathrm{Cdx}^{+/-}$mice. When low CDX2-expressing DLD-1 cells were treated with inhibitors of $\mathrm{mTOR}$, chromosome instability was reduced in a dose-dependent manner, but only if the cells were exposed in the G1-early-S phase of the cell cycle.

Future studies should address what the direct target of CDX2 is that activates mTOR. Confirmation of this pathway of tumorigenesis in human polyposis would provide a rationale for testing mTOR inhibitors for prevention and treatment of cancers that involve chromosomal instability.

Ezzie Hutchinson

\section{(1) References and links}

ORIGINAL RESEARCH PAPER Aoki, K. et al. Colonic polyposis caused by mTOR-

mediated chromosomal instability in $\mathrm{ApC}^{+/ \Delta 716} \mathrm{Cd} \times 2^{+/-}$compound mutant mice. Nature Genet. 16 Nov 2003 (doi: 10.1038/ng1265)

WEB SITE

Makoto Mark Taketo's lab: http://www4.mfour.med.kyoto-v.ac.jp

\section{TRIAL WATCH}

\section{Vaccine victory}

A multi-peptide vaccine causes an antitumour immune response in $80 \%$ of patients with advanced melanoma, according to a randomized, Phase II clinical trial that was conducted by Craig Slingluff and colleagues.

The trial compared two different vaccination approaches against melanoma. Twenty-six patients with stage III or IV melanoma received six vaccinations that consisted of a mixture of four melanoma peptides, plus a tetanus helper peptide and treatment with low-doses of interleukin-2. In 13 patients, the peptides were injected in an emulsion that included the cytokine granulocyte-macrophage colony-stimulating factor (GM-CSF) as an adjuvant. The other 13 patients were injected with monocyte-derived dendritic cells (DCs) that had been pulsed with the peptides.

The authors showed that the immune response against melanoma was greater in the patients who were vaccinated with the GM-CSF emulsion. Antimelanoma cell responses were observed in the lymph nodes that drain the vaccine site in $80 \%$ of patients in the GM-CSF group, but only $13 \%$ of the patients that were vaccinated with peptide-loaded DCs.

Favourable outcomes, including tumour shrinkage, were observed in $31 \%$ of patients who received the GM-CSF-based vaccine, and two patients experienced stable disease for several months. Favourable outcomes were only observed in $15 \%$ of patients in the DC group, and only one patient experienced stable disease. Two patients who received the GM-CSF-containing vaccine also developed vitiligo. The authors suggest that the rate of tumour regression was less than the T-cell response rate because of heterogeneity of tumour-antigen expression and other tumour-escape mechanisms.

ORIGINAL RESEARCH PAPER Slingluff, C. L. Jr et al. Clinical and immunologic results of a randomized phase II trial of vaccination using four melanoma peptides either administered in granulocyte-macrophage colony stimulating factor in adjuvant or pulsed on dendritic cells. J. Clin. Oncol. 21, 4016-4026 (2003)

\section{Aspirin could increase cancer risk}

Taking regular aspirin and other non-steroidal anti-inflammatory drugs (NSAIDs) has been shown to help prevent many diseases, including colorectal cancer. Some animal studies and human data have indicated that aspirin might also inhibit pancreatic carcinogenesis, but analysis of cancer incidence as part of a large prospective study into the risk factors for chronic diseases in women shows that aspirin users actually have an increased risk of developing pancreatic cancer. Eva Schernhammer reported these surprising results at a recent American Association of Cancer Research conference — Frontiers in Cancer Prevention Research.

A total of 88,378 women with no detectable cancer were included in the Nurses' Health Study and were followed up for 18 years. Aspirin use was assessed at baseline and then updated every 2 years. Participants who were taking two or more aspirin tablets a week had a relative risk (RR) for pancreatic cancer of 1.20 compared with non-users. Increasing the duration of aspirin use was also associated with a significantly increased risk: women who had taken aspirin regularly for 20 years or more had a RR of 1.58 . Women who had taken 14 or more aspirin tablets per week for 4 or more years had a RR of 1.86 .

The authors conclude that extended regular use of aspirin is associated with increased risk of pancreatic cancer in women. Other risk factors include smoking and obesity, but taking these factors into account did not alter the association of pancreatic cancer risk with aspirin use. There is some evidence that regular aspirin use might cause pancreatitis - inflammation of the pancreas that has been linked to an increased risk of developing pancreatic cancer - so this connection is worth investigating further.

FURTHER READING Schernhammer, E. et al. A prospective study of aspirin use and risk of pancreatic cancer in women. AACR Proc. Front. Cancer Prev. Res. A162 (2003) 